\title{
"You cannot be serious"
}

\author{
Ivor Slickand
}

It has been suggested that a chaperone may be necessary when general practitioners (GPs) conduct eye examinations, specifically those where the lights need to be dimmed and GPs need to get up close and personal with their patients. Apparently the proximity and heavy breathing into the patient's ear during this type of examination may cause patients alarm. I'd have thought that the caffeine-fuelled shaking of the piercing beam of light from the ophthalmoscope would have been enough to achieve this.

Just how far will this madness be allowed to go? Touching a person on the arm may be interpreted by some as invading their personal space or as being intimate, so presumably innocent activities such as measuring blood pressure or taking blood samples will in future mean 'let's get the chaperone in'. Am I going to have to abandon the economic and environmentally friendly, low-energy, but rather dim, consultation room light bulb too, and revert back to the traditional in-your-face, 100-watt, bad-for-theenvironment one as well then? Will I have to stop having relaxing classical music in my consultation room too if I'm to avoid being accused of trying to seduce my patients?

Surely the answer here is not a chaperone (who in the dimly lit room wouldn't see whether the GP was having a quick grope anyway) but a clear explanation to the patient about what the examination involves and why it is being performed.

But it's not only doctors who will need chaperones. It follows that practice nurses will need them too as they take on more responsibility. It's going to become like a theatrical farce, with GPs and nurses spending the bulk of their clinic time running from room to room. As one GP runs to chaperone the nurse, another GP will be racing to assist a colleague, whilst the practice nurse, or nurses, run(s) along to chaperone one of the GPs. Unlike on the stage though, at least it should only be the patient who is in a state of undress.

Even if enough chaperones are available in general practice - and there won't be - what about home visits? Many examinations are done with patients on the sofa or on their bed. Here more than anywhere else a chaperone is needed. Reason enough to abolish home visits perhaps.

If quality framework points were attached to chaperone attendance it might be a more attractive option - sorry, I meant to say a more 'beneficial to the patient' option, I didn't mean attractive, honestly. Not even humour will be allowed during a consultation. Well, according to a recent study from Wales, if this is the case then men will really find it hard to open up and talk with their doctor about their health concerns. So that's all the work encouraging men to come to see their doctor down the drain then.

But the solution, apparently, is simple. There will become available an army of trained chaperones introduced into the National Health Service (NHS) - the 'watchers' as they will come to be known. Yeah right. The NHS can't recruit enough people as it is because of the lousy conditions and pay, so who's going to volunteer to train as a chaperone? Probably those individuals who shouldn't be anywhere near vulnerable patients, those who have become

J Fam Plann Reprod Health Care 2005; 31(1): 78

Touchingswell, UK

Ivor Slickand, General Practitioner bored looking at similar images on the Internet and now want a reality that isn't virtual.

But clearly there is something even more sinister going on. These trained chaperones are the ultimate GP assessment tool as they covertly observe a GP's knowledge and ability to practice. We've had videos for a long time for GP assessment; but as people have now come to realise, simple home computer technology enables video consultations to be edited and re-voiced to standards that make them as likely to qualify for a BAFTA as the MRCGP.

But the introduction of trained chaperones may provide us with a valuable opportunity that should not be missed.

For a while now I have dreamt of having a personal assistant with me when I'm consulting. You know, someone who sits in on the consultation, and digs around for necessary forms when needed, fills them in, knows the exact Read code for the diagnosis I make, and holds on the line whilst the local hospital tries to track down the on-call registrar. The mundane but necessary tasks that take up so much of a GP's time. Perhaps this role and the role of chaperone could be combined?

Let's face it, how often do GPs actually examine patients anyway? Instead of having these chaperones sitting twiddling their thumbs, let's train them to perform other tasks for us whilst they're waiting to put their chaperoning skills into practice. This will avoid us wasting time trying to find a chaperone when we need one, or having to arrange for a second appointment when a chaperone can't be found, because they'll be there next to us all the time. It makes sense, since if health care professionals are to be protected in this increasingly litigious world, and if their defence organisations are going to continue to protect them, then sadly the bottom line is that in future chaperones are going to have to accompany the GP everywhere anyway. As a bonus, patients will come to accept and understand that having a chaperone in the room is the norm rather than 'it must be because my doctor is a pervert'.

Oh no, I've gone and written this without a chaperone present. Hopefully my computer won't misinterpret the way I've been prodding at its keyboard...

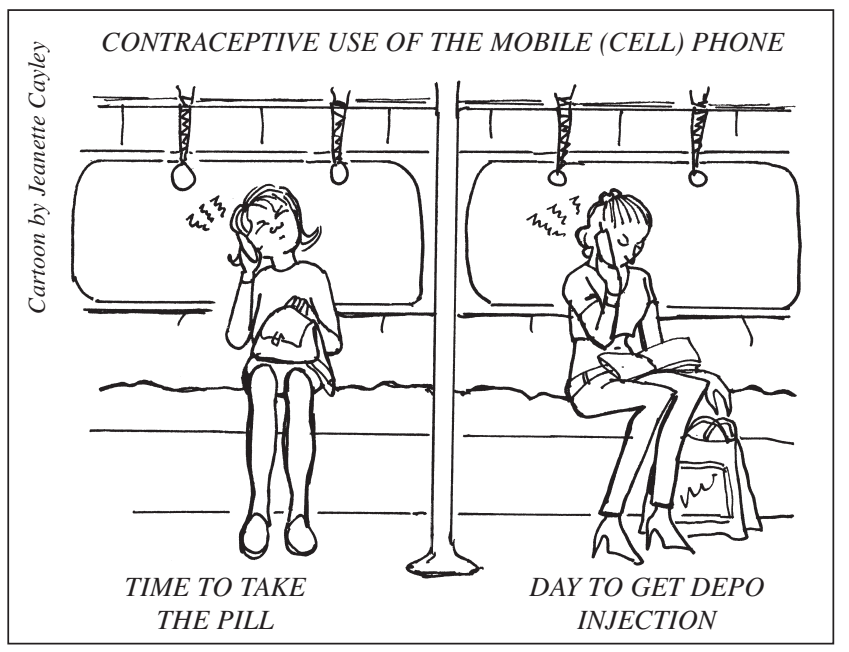

